\title{
Reversible acute renal failure induced by losartan in a renal transplant recipient
}

\author{
M Ostermann, DJA Goldsmith, T Doyle, JC Kingswood, P Sharpstone
}

\begin{abstract}
Summary
A 56-year-old man who received a liverelated renal transplant in 1988 was started in 1995 on the selective angiotensin II antagonist losartan (Dupont-Merke) to treat worsening hypertension. Two months later because of pulmonary oedema, loop diuretics were started. Within two weeks, serum creatinine had increased from 245 to $571 \mu \mathrm{mol} / 1$, and the patient became oliguric. A systolic bruit was noted over the graft. Renal angiography showed a $\mathbf{9 0 \%}$ stenosis of the transplant renal artery. Losartan was withdrawn, with prompt improvement in renal function. A successful percutaneous transluminal angioplasty performed a few days later resulted in further improvement in renal function accompanied by a significant diuresis.
\end{abstract}

Keywords: renal failure, transplant, angiotensin, losartan

Indications for the use of angiotensin-converting enzyme (ACE) inhibitors are increasing. These include hypertension, heart failure, myocardial infarction, diabetic nephropathy, and proteinuric renal disease. ACE inhibitors have several neurohumoural actions beyond blockade of the renin-angiotensin system; in particular, the potentiation of bradykinin is considered responsible for some of their sideeffects. $^{1}$

Renal problems with ACE inhibitors have been noted from their inception. In one recent report, $16 \%$ of cases of acute renal failure were attributed to the use of ACE inhibitors. ${ }^{2}$ Most commonly, where there is critical renovascular disease, glomerular filtration is severely reduced in the presence of ACE inhibitors, as efferent renal arteriolar tone, upon which infiltration then depends, is exquisitely dependent on angiotensin II. Though most investigators invoke this angiotensin mechanism to explain deterioration in renal function after the use of ACE inhibitors, there has been speculation that bradykinin may also be relevant. ${ }^{1}$

In part because of these side-effects, and from pharmacological considerations, research has focused on producing selective antagonists of angiotensin II, at the level of the cellular angiotensin AT1 receptor. ${ }^{1}$ The prototype of this new class of drug is losartan. We report a case of acute renal failure in a renal transplant recipient given losartan followed by loop diuretics, for increasingly severe hypertension and pulmonary oedema.

\section{Case report}

The patient was born in 1939. In 1977 proteinuria was noted. In 1986 he developed nephrotic syndrome and hypertension caused by diffuse proliferative glomerulonephritis with focal sclerosis. Renal function declined rapidly, despite control of blood pressure.

Before becoming dialysis-dependent the patient underwent live-related renal transplantation in late 1988 (donation from his brother). The donor artery was spatulated end-to-end with the recipient internal iliac artery. Rejection was fierce, and required pulsed methylprednisolone, and three courses of anti- $T$ cell therapy using ATG and OKT3, all within a few months after engraftment. An early renal transplant angiogram showed a minor stenosis at the origin of the internal iliac artery.

Transplant function one year after transplantation was fairly good with a plasma creatinine of around $200 \mu \mathrm{mol} / 1$ on maintenance immunosuppression of prednisolone, azathioprine and cyclosporin A. Hypertension continued to require drug therapy after renal transplantation. Post-transplant erythrocytosis developed four years after transplantation and required venesection. An isotope renogram at this time showed good renal perfusion and prompt excretion.

In 1994, the patient developed increasing leg oedema and a further rise in blood pressure. Amlodipine was stopped, and enalapril started at $5 \mathrm{mg}$ (later $10 \mathrm{mg}$ ) daily. Over the first two months of enalapril therapy serum creatinine was stable (figure 1). The patient noticed intermittent claudication, and femoral bruits were found on examination later in 1994. A Doppler ultrasound of the renal transplant in 1994 revealed poor perfusion and damping of renal artery Doppler signals.

Early in 1995, enalapril was stopped by the patient because of tiredness, without a subsequent change in renal function (figure 1). Over the next few months hypertension worsened, and in August 1995, with a plasma creatinine of $245 \mu \mathrm{mol} / \mathrm{l}$, the patient started losartan $50 \mathrm{mg}$ daily. Two months later the patient had sudden pulmonary oedema, with no evidence of myocardial ischaemia or infarction, and was admitted to start intravenous, then oral, diuretics. Plasma creatinine increased 
from its previous stable baseline over the next few days as his pulmonary oedema resolved, and his weight and blood pressure fell. Another Doppler ultrasound of the renal transplant showed a poor perfusion pattern, with arterial signal damping and no evidence of renal arterial stenosis at or near the site of the vascular anastomosis, which was apparently well seen. Losartan was increased to $100 \mathrm{mg}$ to treat recurrent hypertension, and over the next 11 days the patient became oliguric and his plasma creatinine rose sharply (figure 1).

A further Doppler study of the graft showed a normal arterial waveform and a normal intrarenal resistive index. A selective renal angiogram performed a day later showed a tight $(90 \%)$ stenosis at the origin of the transplanted renal artery with post-stenotic dilatation and normal intrarenal arteries (figure 2). A transplant renal bruit was now audible.

Losartan was stopped immediately, and doxazosin used to control blood pressure. Within two weeks the creatinine fell to the previous baseline (figure 1). A successful percutaneous renal transplant angioplasty resulted in sharp falls in creatinine and blood pressure, and polyuria (6 $1 /$ day). The patient is now free from breathlessness, has a normal blood pressure on no antihypertensive drugs, no renal transplant bruit, and a creatinine lower than at any time post-engraftment.

\section{Discussion}

This case encapsulates all the salient points about renovascular disease, and is the first to describe the theorectically predictable occurrence of acute renal failure after blockade of the action of angiotensin II by the specific angiotensin antagonist losartan (see Note added in proof).

It is probable that renovascular disease in our patient had been slowly developing for some time, with progressively but slowly rising blood pressure, and a modest but initially nonprogressive rise in creatinine. ACE inhibition in the absence of diuretics had not changed

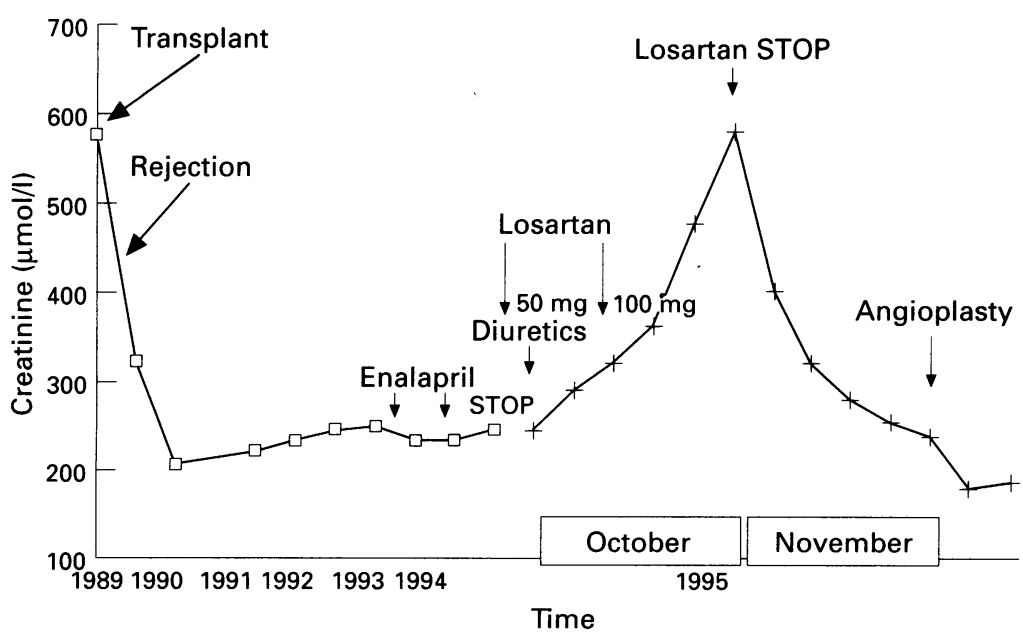

Figure 1 Plot of plasma creatinine against time showing the effect of losartan on renal function

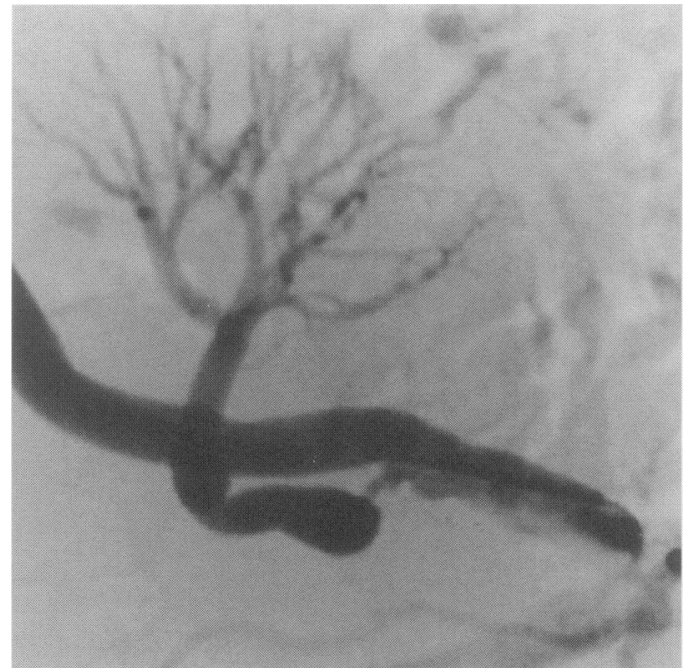

Figure 2 Renal angiogram showing stenosis at the origin of the transplanted renal artery

\section{Learning points}

Renovascular disease is commoner in men, smokers, with hyperlipidaemia and atheroma elsewhere (eg, carotid, coronary, ilio-femoral); it also increases with age

excretory renal function. Renovascular disease has been shown to be more common in renal transplantation where there has been severe early rejection (W Wong, personal communication), as in this case. Hypertension and sudden pulmonary oedema are classical, though of course not specific, signs of renovascular disease. ${ }^{3,4}$ The patient also had evidence of vasculopathy elsewhere (femoral bruits); again, atherosclerotic renal arterial disease is commoner in patients with widespread atherosclerosis from hyperlipidaemia, diabetes or smoking. ${ }^{3,4}$ Diuretics are wellknown to potentiate the action of drugs that block the renin - angiotensin system, as volume depletion is a prime stimulus for renin release and is a situation where blood pressure is more dependent on angiotensin-induced arteriolar vasoconstriction (hence the practice of reducing the strength of contemporaneously administered diuretics when starting ACE inhibitors to ameliorate otherwise troublesome postural hypotension).

This case also demonstrates that the investigation of choice in making the diagnosis of renovascular disease remains renal angiography, as alternative, less invasive, techniques such as Doppler ultrasonography have lower sensitivities and selectivities.

In conclusion, losartan will no doubt prove to have a useful place in the therapeutic armamentarium, at least as an alternative to ACE inhibitors where side-effects limit their use, but vigilance must be exercised in clinical conditions where renovascular, or hypertensive renal, disease has produced a fixed renal arterial blood supply dependent on angiotensin II. For practical purposes, renal function should be measured sufficiently often after 
initiation of therapy with losartan, or after potentiation of its effects by increasing contemporaneous diuretics, to detect a deterioration in renal function at an early stage before acute renal failure is established and dialysis required.

1 Johnston CI. Angiotensin receptor antagonists: focus on Losartan. Lancet 1995; 346: 1403-7.

2 Ritz E, Koch $M$. Renal failure in diabetic neuropathy. In: Cameron S, Davison AM, Grunfeld J-P, Kerr D, Ritz E, eds. Oxford textbook of clinical nephrology. Oxford: Oxford

3 Mann J, Allenberg J-R, Reisch C, et al. Renovascular Mann J, Allenberg J-R, Reisch C, et al. Renovascular
hypertension. In: Cameron S, Davison AM, Grunfeld J-P, hypertension. In: Cameron S, Davison AM, Grunfeld J-P,
Kerr D, Ritz E, eds. Oxford textbook of clinical nephrology. Kerr D, Ritz E, eds. Oxford textbook of clinical nephrol.
Oxford: Oxford University Press, 1992; pp 2096-117.
Note added in proof: Another case of losartan-induced azotaemia has now appeared in print. $^{5}$ It concerns a diabetic with no angiographic signs of transplant renal artery stenosis.

4 Breyer JA, Jacobson HR. Ischaemic renal disease. In Cameron S, Davison AM, Grunfeld J-P, Kerr D, Ritz E eds. Oxford textbook of clinical nephrology. Oxford: Oxford University Press, 1992; pp 1077-84.

5 Cohen LS, Friedman EA. Losartan-induced azotemia in diabetic recipient of a kidney transplant. N Engl f Med 1996; 354: 1271 .

\title{
Spontaneous contralateral pneumothorax following pneumonectomy
}

\author{
JGH Hubbard, UU Nkere, NK Bhatnagar
}

\begin{abstract}
Summary
A case of a spontaneous pneumothorax occurring three days post-pneumonectomy is presented. Difficulties in diagnosis and management are discussed.
\end{abstract}

Keywords: pneumonectomy, spontaneous contralateral pneumothorax

We report on a rare complication following pneumonectomy with potentially fatal consequences.

\section{Case report}

A 65-year-old man underwent a routine left pneumonectomy for squamous cell carcinoma. His early post-operative recovery was uneventful. His post-operative chest X-ray demonstrated satisfactory accumulation of fluid in his pneumonectomy space and good expansion of his remaining lung (figure 1). On the third post-operative day, while undergoing chest physiotherapy, he suddenly developed a cough productive of copious, frothy, blood-stained sputum and became short of breath.

On initial examination he was found to be sweaty with a pulse of 100 beats $/ \mathrm{min}$, respiratory rate 30 breaths/min, blood pressure 179/ $100 \mathrm{mmHg}$ and oxygen saturation $96 \%$. He became increasingly unwell and his oxygen saturation dropped to $77 \%$. His jugular venous pressure was noted to be elevated on the right and he had reduced air entry on this side. A chest X-ray performed on the ward within minutes confirmed the clinical findings of a right pneumothorax (figure 2), the fluid level in his pneumonectomy space was unchanged from that mornings X-ray. He immediately improved following insertion of a right-sided chest drain. Twenty-four hours later he developed subcutaneous emphysema, therefore a bronchoscopy was performed, demonstrating a healthy intact bronchial stump on the left side and a normal right bronchial tree. His subcutaneous emphysema gradually settled. Tetracycline pleurodesis was performed on three consecutive days. The chest drain was subsequently removed and chest X-ray showed no residual pneumothorax. He was discharged home on his ninth post-operative day.

\section{Discussion}

Contralateral pneumothorax following pneumonectomy is rare. Blalock reported four cases complicating 340 pneumonectomies and Har-

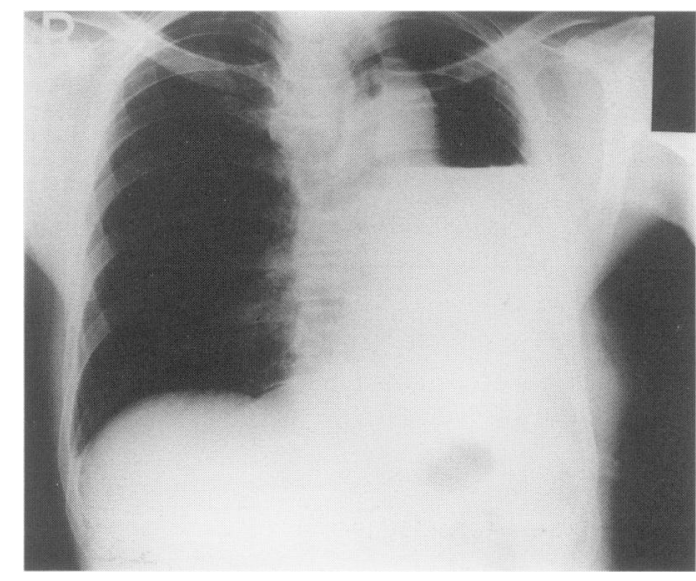

Figure 1 Chest $\mathrm{X}$-ray on the morning of the third post-operative day 